\title{
Análisis de la Contaminación por flujo vehicular en un entorno universitario
}

\section{Analysis of pollution by vehicular flow in a university environment}

\section{Ligia Beleño Montagut.; Juan Diego Colegial Gutierrez²}

\author{
${ }^{1}$ Docente de tiempo completo. Universidad Autónoma de Bucaramanga, Departamento de \\ Matemáticas y Ciencias Naturales. lbeleno@unab.edu.co \\ 2. Docente de tiempo completo. Universidad Industrial de Santander. Facultad de Ingenierías Físico \\ Químicas, Escuela de Geología. colegial@uis.edu.co
}

\section{Resumen}

En Colombia se han realizado estudios de monitoreo ambiental, los cuales han permitido conocer el estado de contaminación de algunas ciudades, dentro de los cuales se destacan aportes de trabajos investigativos asociados a efectos sobre la salud humana y el medio ambiente. Este trabajo forma parte de un proyecto de investigación sobre la contaminación ambiental en cercanías a la Universidad Autónoma de Bucaramanga y dado que no hay datos relacionados en esta zona se realizaron las mediciones de concentración de material particulado $\mathrm{PM}_{2.5}, \mathrm{COV}_{\mathrm{s}_{1}}$ humedad relativa $\mathrm{y}$ temperatura en distintas franjas horarias durante 26 días para establecer un registro base. Adicionalmente se realizaron mediciones de tráfico vehicular en las mismas franjas y fechas. Como resultado del análisis se encontró que hay una diferencia significativa de las emisiones medidas respecto a las presentadas en el "día sin carro" y que los factores meteorológicos influyen en los valores de concentración obtenidos. El artículo permite proyectar un escenario que puede ser tomado como referente para posteriores estudios por autoridades interesadas tanto en el análisis del impacto de la contaminación causadas por el tránsito vehicular como en aquellas interesadas en implementar controles a emisiones de los vehículos.

Palabras clave: calidad del aire, material particulado, compuestos orgánicos volátiles.

\section{Abstract}

In Colombia several environmental monitoring studies have been carried out, which have allowed to know the pollution status of some cities, among which contributions of research works associated with effects on human health and the environment stand out. This work is part of a research project on the environmental 
pollution in surroundings to Universidad Autonoma de Bucaramanga and since there are no related data in this area were made the PM2.5 particulate material concentration measurements, VOCs, relative humidity and temperature in different time slots for 26 days to establish a base register. Additionally, measurements of vehicular traffic were made in the same time zones and dates.

As a result of the analysis, it was found that there is a significant difference in the emissions for the non-car day and that the meteorological factors influence the

\section{Introducción}

La contaminación ambiental atmosférica es un problema que merece mayor atención de la que actualmente tiene, un ejemplo de esta situación es la escasa o carente reglamentación; que en algunos países existe respecto a ciertas especies químicas consideradas como nocivas para la salud y el ambiente. La normativa ambiental vigente se encuentra fundamentada en el Decreto 948 de 1995 (Ministerio del Medio Ambiente, 1995), las Resoluciones 610 de 2010 (Ministerio de Ambiente, Vivienda y Desarrollo Territorial., 2010), 1541 de noviembre de 2013 (Ministerio de Ambiente, Vivienda y Desarrollo Territorial., 2013), en las cuales se establecen los niveles de calidad de aire y el procedimiento para la evaluación de actividades que generan olores ofensivos; recientemente, la Resolución 2254 de noviembre de 2017 (Ministerio de Ambiente, 2017) estableció
29

concentration values obtained. The study allows to project a stage that can be taken as a reference for further studies and for the authorities in charge of traffic analysis interested in implementing vehicle emissions controls.

Keywords: air quality, particulate matter, Volatile Organic Compounds

un ajuste progresivo en cuanto a los máximos permitidos de contaminantes criterio, incluye nuevos contaminantes y define elementos técnicos para gestionar la calidad del aire.Las redes de monitoreo se clasifican en automáticas, manuales o mixtas; en Colombia solamente 7 de las 20 redes son automáticas (IDEAM, 2012). En el caso particular de la ciudad de Bucaramanga, por una parte no todas las estaciones miden los mismos contaminantes y por otra, la estación más cercana a la Universidad Autónoma de Bucaramanga (zona de estudio) está ubicada a más de 700 metros en la zona de cabecera del llano.Una de las mediciones de las redes de monitoreo es el material particulado (Partículas suspendidas totales y PM10), el cual ha sido definido como contaminante criterio, al ser identificado como común en la atmósfera además de 
30

ser perjudicial para la salud (IDEAM, 2007).De acuerdo al Informe Nacional de Calidad Urbana de 2015 (Ministerio de Ambiente y Desarrollo Sostenible, 2015) , Bucaramanga se encuentra en el rango de calidad baja según los reportes de índice de calidad ambiental urbana-ICAU, 2013.

Este artículo sintetiza el desarrollo de la investigación realizada en inmediaciones de la Universidad Autónoma de Bucaramanga, en la cual se plantea y analiza una base de mediciones de concentración para el PM2,5, COV y algunos parámetros meteorológicos.

\section{Metodología}

\subsection{Datos y zona de estudio}

Se realizaron mediciones tales como la humedad relativa, PM2.5 (partículas con diámetro menor a $2.5 \mu \mathrm{m}$ ) y CVO (Compuestos Orgánicos Volátiles) mediante un Multímetro de Calidad de aire con las siguientes características:

PM2.5: Rango: 0-500 $\mu \mathrm{g} / \mathrm{m}^{3}$; precisión: \pm 10\%; ciclo de muestreo: 1 segundo.

VOC: rango: 0-10 ppm; precisión: \pm 10\%; ciclo de muestreo: 1 segundo.

Temperatura: Rango: $-10-80^{\circ} \mathrm{C}$ precisión: $\pm 0.3^{\circ} \mathrm{C}$; ciclo de muestreo: 2 segundo.
Humedad: Rango: 0-100\% RH; precisión: $\pm 3 \% \mathrm{RH}$; ciclo de muestreo: 2 segundo.

Al mismo tiempo del muestreo manual se realizaron los aforos vehiculares de acuerdo a la siguiente clasificación: automóviles, buses, vehículos pesados y motos, cada 5 minutos en las horas de mayor flujo vehicular: 7-8 horas, 12-13 horas y 18-19 horas. El tiempo durante el cual se tomaron las mediciones fue de lunes a sábado durante 26 días. Para los datos meteorológicos de velocidad y dirección del viento se tomaron como referencia los promedios reportados en el informe (IDEAM, 2012) para la estación más cercana.

\section{Resultados}

\subsection{Aforo vehicular}

En las gráficas de la fig. 1 que relacionan la cantidad de vehículos promedio y se observa una diferencia significativa en el "día sin carro" (día 2 en la gráfica) y en el lunes festivo (día 22 en la gráfica), respecto a los demás días. También se observa que el número de automóviles (entre 200 y 800 en dichas franjas) supera el doble de la cantidad de motos. El día de menor circulación es el lunes festivo siendo $54,5 \%$ correspondiente a vehículos particulares y taxis, 38,8\% motocicletas y $4,3 \%$ buses y vehículos pesados.

\subsection{Medida de PM2.5}


31

De acuerdo a la fig. 2, las concentraciones de PM 2.5 para la avenida 42 de todos los vehículos que transitan por la vía durante el periodo de medición muestra valores cercanos en las concentraciones siendo los valores más bajos 39,5 $\mathrm{\mu g} / \mathrm{m}^{3}$ (valor en el "día sin carro"), y 40,1 $\mu \mathrm{g} / \mathrm{m}^{3}$ (día lunes festivo), en general más bajos en la mañana y más altos en la noche. El promedio total es de $43,9 \mu \mathrm{g} / \mathrm{m}^{3}$ con una correlación de 0,94 entre la franja mañana y tarde, 0,86 entre la franja mañana y noche y 0,85 entre tarde y noche.

\subsection{Medida de COV}

De acuerdo con la fig. 2, las concentraciones de $\mathrm{COV}$, que corresponden en esencia a hidrocarburos en estado gaseoso a la temperatura ambiente y muy volátiles a dicha temperatura, tienen valores más bajos en el "día sin carro" con un valor de 0,088 ppm y 0,1 ppm el lunes festivo con respecto a los demás días de medición, siendo el promedio total de 0,41 ppm, con una correlación de 0,88 entre la franja mañana y tarde, 0,56 entre la franja mañana y noche y 0,58 entre tarde y noche.

\subsection{Medida de la Humedad Relativa}

La humedad relativa representa la relación entre la cantidad de vapor de agua que contienen el aire y la cantidad necesaria para alcanzar la saturación a la misma temperatura.
Con relación a la humedad relativa promedio se identifican mínimos coincidentes respecto al $\mathrm{PM} 2,5$ en el lunes festivo, pero no es muy notorio en el día del no carro. (Ver fig. 3.). En cuanto al promedio presenta valores entre $60 \%$ y $70 \%$ siendo los más bajos al medio día.

\subsection{Medida de la temperatura}

La diferencia es de $2{ }^{\circ} \mathrm{C}$ en la temperatura promedio de la franja horaria del medio día respecto a las otras dos franjas, con variaciones mínimas (Ver fig. 4). La temperatura promedio es de 28 ${ }^{\circ} \mathrm{C}$ en la mañana y en la noche, y de $31^{\circ} \mathrm{C}$ al medio día.

\subsection{Análisis de la dirección y velocidad del viento}

A partir de los datos meteorológicos registrados en el informe (IDEAM, 2012) respecto al promedio multianual (20052009), la velocidad de los vientos en Neomundo (estación meteorológica más cercana) para los meses septiembre y octubre muestran gran similaridad tanto en magnitud como dirección siendo ésta última procedente del NE. Adicionalmente, en la franja del medio día son ligeramente mayores las magnitudes de velocidad pero en la tarde disminuye nuevamente.

Durante las fechas de la medición, se observa un ligero incremento en cuanto al PM2.5 en octubre, y un incremento significativo en el COV. Lo anterior a pesar de las lluvias en el mes de octubre dado que de acuerdo a reportes 
32

históricos del clima, en Bucaramanga se presenta la mayor época de lluvia en el mes de octubre. Un factor significativo que permite explicar tal comportamiento obedece a que se presentan vientos débiles en la zona por lo cual los contaminantes pueden acumularse alrededor de la fuente de emisión (Punto de medición de la avenida 42). Por otra parte la inversión térmica representa otro factor importante en cuanto al comportamiento de la contaminación.

En concordancia con (GrupoTIPSA, 2009) la influencia de la magnitud de la velocidad y su dirección se convierten en un problema de mayor complejidad el cual será abordado en otra fase de este proyecto de investigación.

\subsection{Flujo vehicular, variables meteorológicas y contaminante}

Se hicieron las mediciones respectivas en el punto de mayor concentración correspondiente a la parada de bus sabiendo que la mayor liberación de contaminantes se produce en el momento de arranque.

Las gráficas 5 y 6 representan el número de vehículos de cada clase (buses, automóviles, motocicletas). Se identifica mayor tráfico en el día jueves y menor en el día sábado.

Con relación a las concentraciones de PM2.5 para la avenida 42 de todos los vehículos que transitan por la vía durante el periodo de medición muestran valores entre $41,6 \mu \mathrm{g} / \mathrm{m}^{3}$ (valor promedio del día lunes en la mañana) y $45,48 \mu \mathrm{g} / \mathrm{m}^{3}$ (valor promedio del miércoles en la noche), siendo en general más bajos en la mañana y más altos en la noche.

Se observa que los días sábado disminuye el número de vehículos que transita por la avenida 42 y de manera concordante también disminuyen los parámetros $\mathrm{PM}_{2,5}$ y el COV medidos.

Es importante resaltar que aun cuando no se cuente con una estación fija de monitoreo, los estudios de tráfico vehicular en zonas estudiantiles permiten identificar un estado base acerca de la contaminación en la zona útil para posteriores estudios. Un trabajo similar fue desarrollado en Villa del Rosario (Norte de Santander) en el año 2012 donde se realizó el monitoreo de la fracción respirable PM2.5con resultados que sobrepasan la norma de la OMS de dicho contaminante, concluyendo que está asociado fundamentalmente a la combustión vehicular (Quijano P, 2013).

La Tabla 1. Relaciona las correlaciones obtenidas entre las variables analizadas. Se encontró que cuando la correlación entre la humedad relativa y la temperatura es mayor (que corresponde al medio día, y muestra la relación inversa entre dichas variables), las demás variables tienen mayor factor de correlación.

Tabla 1. Correlación entre variables

\begin{tabular}{|l|r|r|l|l|}
\hline & PM2,5 & COV & HR & T \\
\hline PM2,5 & $\mathbf{0 , 9 4}$ & & & \\
\hline COV & 0,64 & $\mathbf{0 , 8 8}$ & & \\
\hline HR & 0,42 & 0,49 & $\mathbf{0 , 4}$ & \\
\hline T & $-0,54$ & $-0,5$ & $-0,78$ & $\mathbf{0 , 5 5}$ \\
\hline
\end{tabular}

\section{Conclusiones}


El presente estudio describe la incidencia de características meteorológicas en el fenómeno de la contaminación para el caso particular de la avenida 42 con calle 48 de Bucaramanga.

Esta medición inicial que se hizo en la UNAB permitirá tener una base importante para analizar el entorno ambiental en la universidad mientras las entidades competentes actualizan su sistema de medición.

El resultado será relevante para la institución ya que las emisiones del tráfico vehicular contribuyen como principal foco contaminante y a la fecha no hay registro de mediciones ambientales relacionadas, por lo cual se propone hacer un seguimiento anual que permita en dado caso tomar medidas al respecto.

\section{Referencias Bibliogràficas}

GrupoTIPSA. (Junio de 2009). Estudio predictivo sobre inmisiones, del nuevo centro de transportes de interés autonómico(CTIA) de Bailén. Obtenido de https://www.juntadeandalucia.es/medio ambiente/portal_web/ot_urbanismo/urba nismo/planeamiento/planes_iniciativa_c onsejeria/pdf_mercancias/eia_anexo_2.p df

IDEAM. (2007). Informe Anual sobre el Estado del Medio Ambiente y los Recursos Naturales Renovables en Colombia: Calidad del Aire1. Bogotá: http://documentacion.ideam.gov.co/open
biblio/bvirtual/020650/CalidaddelAireC apitulo1.pdf.

IDEAM. (2012). Estado de la Calidad del Aire en Colombia 2007-2010. Bogotá.

Ministerio de Ambiente. (2017). Resolución 2255 de 2017.

Ministerio de Ambiente y Desarrollo Sostenible. (2015). Informe Nacional de Calidad Urbana.

http://capacitacion.siac.ideam.gov.co/SI AC/INFORME_CALIDAD_AMBIENT AL_URBANA.pdf. 
34

Ministerio de Ambiente, Vivienda y Desarrollo Territorial. (2010). Resolución $0610 \mathrm{del}$ 24 de marzo de 2010. Bogotá.

Ministerio de Ambiente, Vivienda y Desarrollo Territorial. (2013). Resoluciòn 1541 de 2013.
Ministerio del Medio Ambiente. (1995). Decreto 948 de 1995.

Quijano P, A. M. (2013). : 2013.11 (2):40-54. Determinación de Metales y Material. Bistua Revista de la Facultad de Ciencias Basicas., 40-54. 

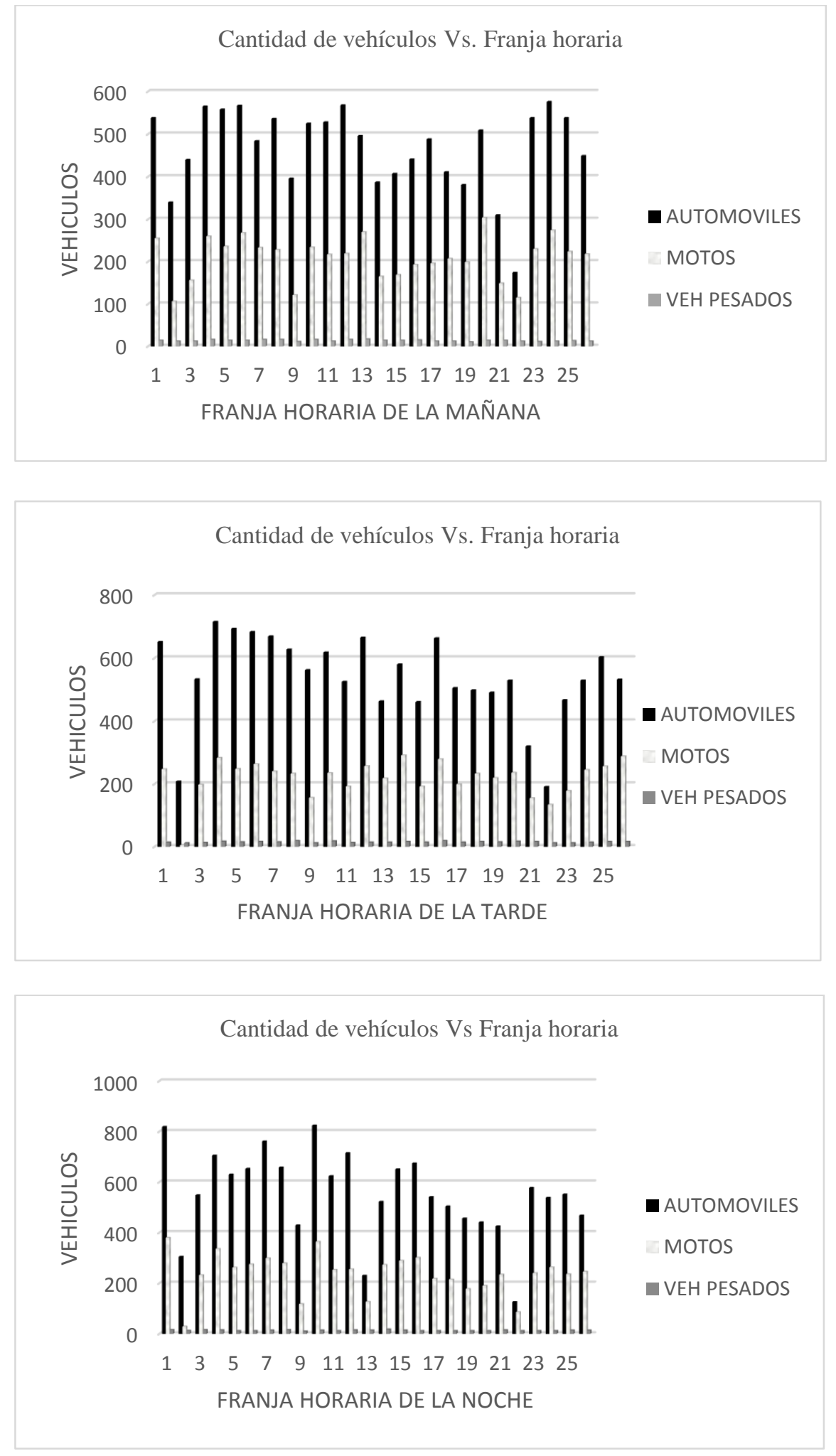

Figura. 1. Aforo vehicular para las franjas: Día, tarde y noche 

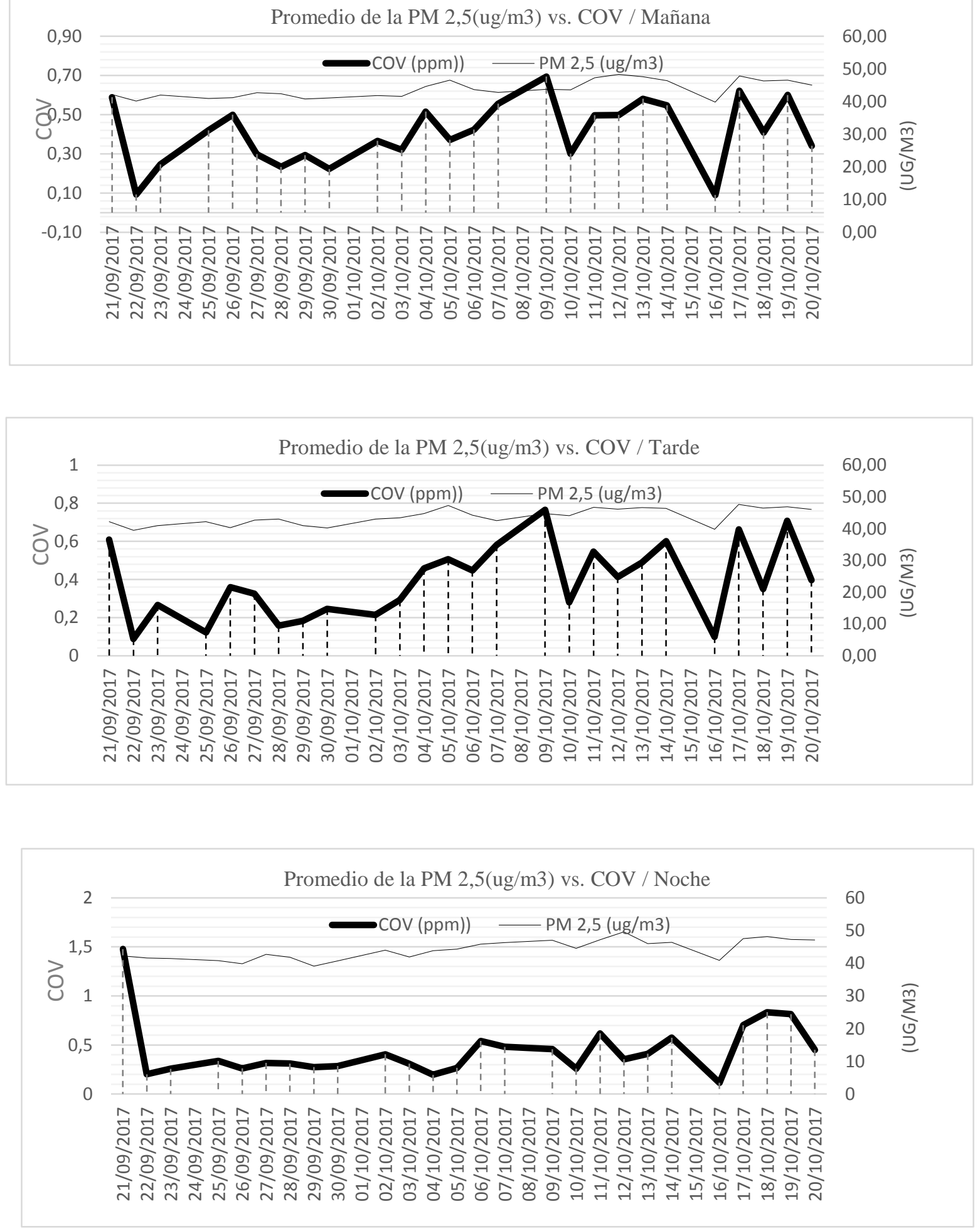
37

Figura 2. Medida de PM2.5 y COV para las franjas: Día, tarde y noche
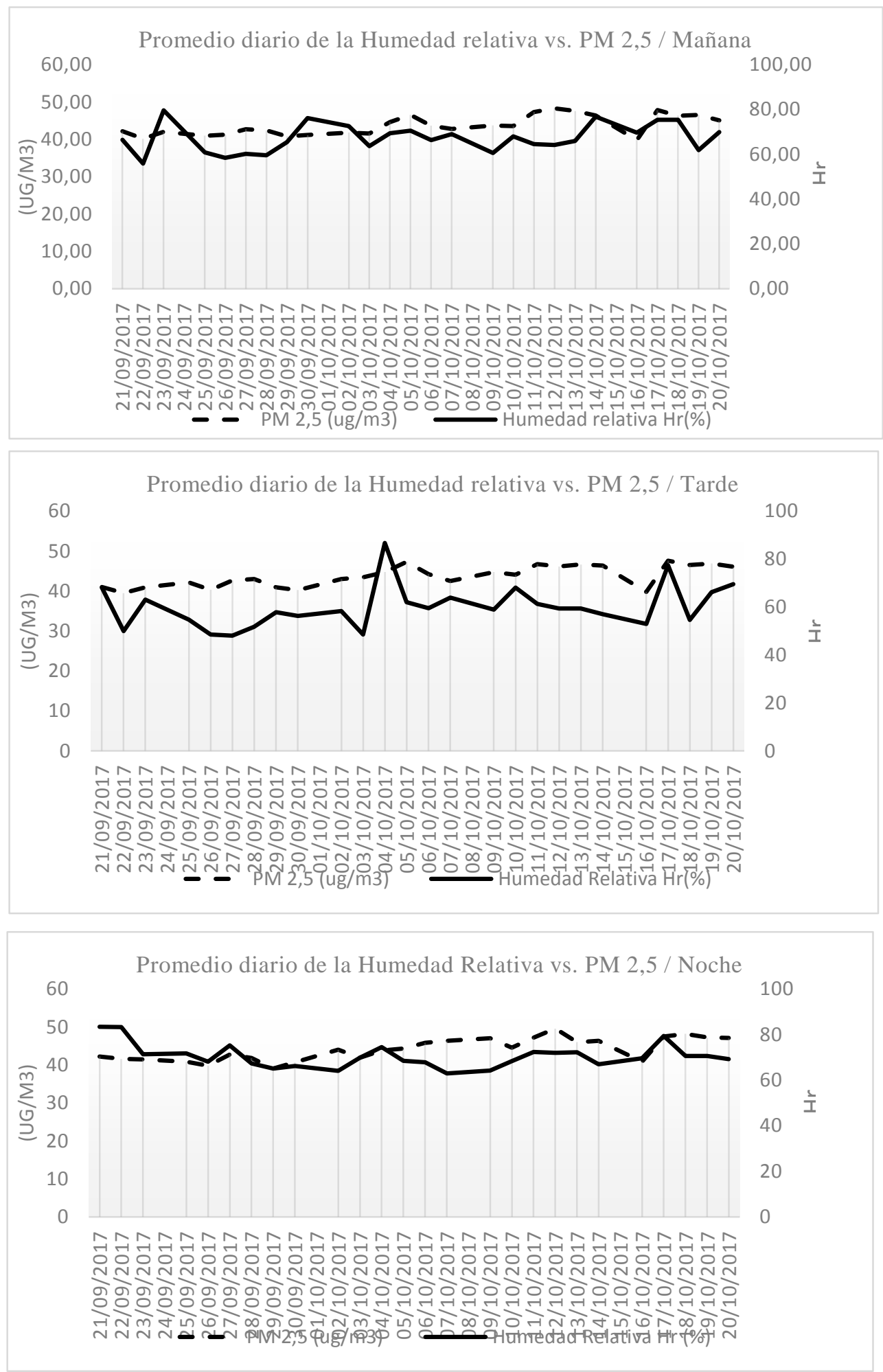
Figura 3. Medida promedio de la humedad Relativa y la concentración de PM2,5 para las franjas: mañana, tarde y noche

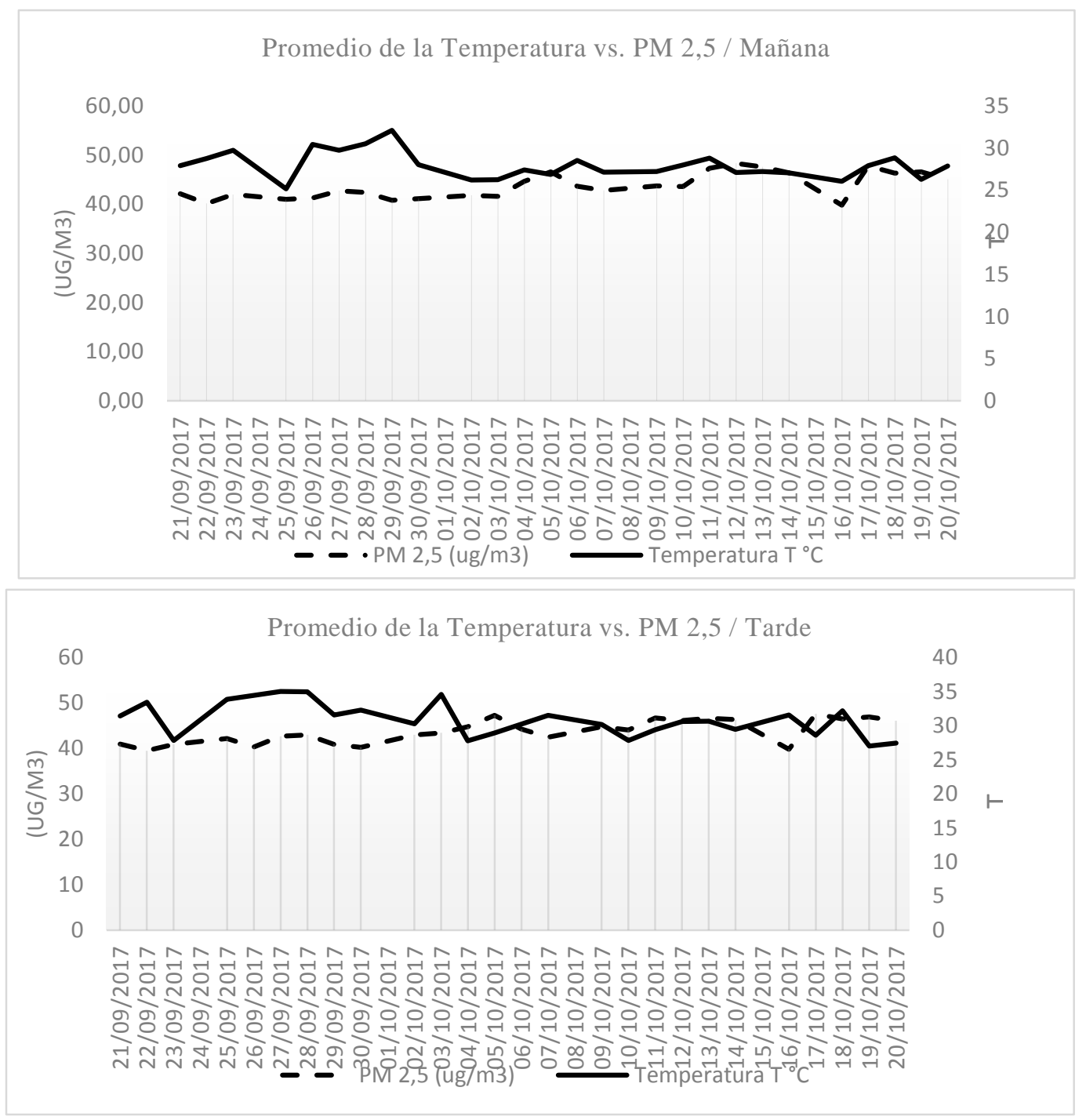


39

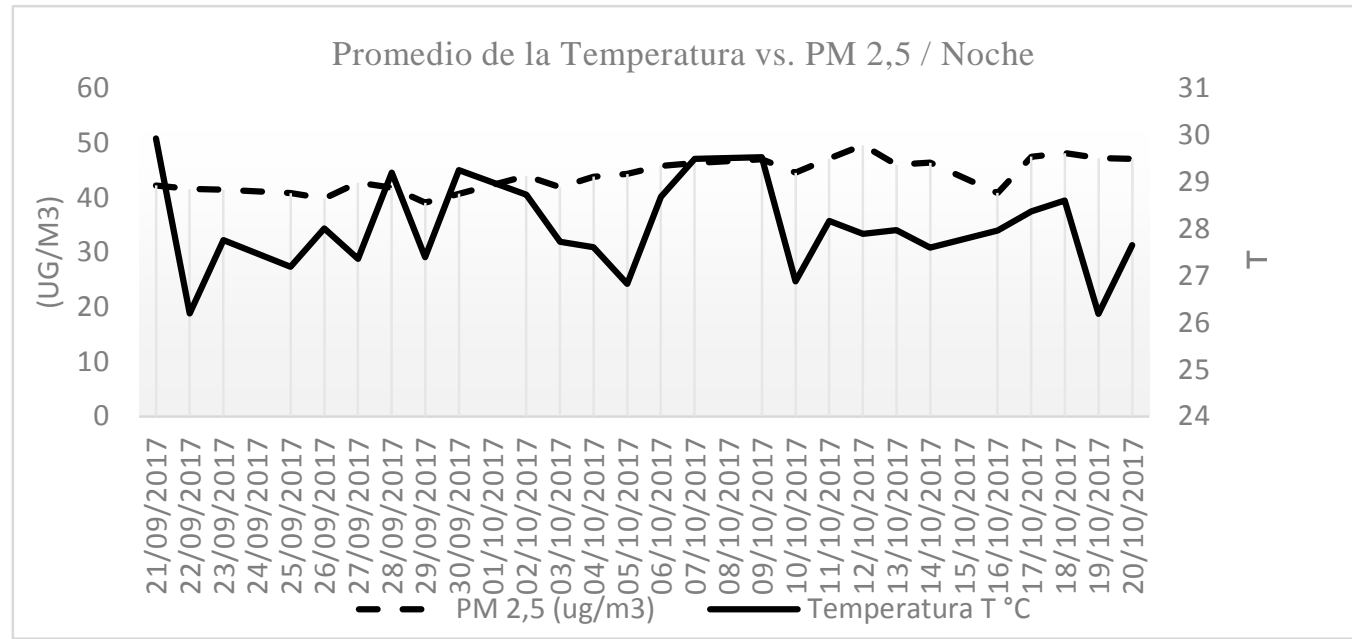

Figura 4. Medida promedio de la Temperatura y la concentración de PM2,5 para las franjas: Día, tarde y noche

Flujo vehicular y Concentraciones de PM 2,5 promedio en la franjas horarias por hora de los dias Lunes

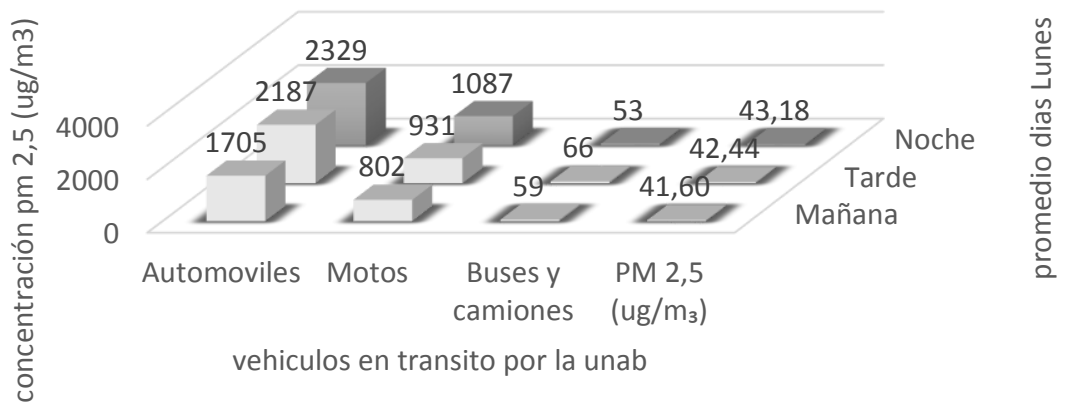

Flujo vehicular y Concentraciones de PM 2,5 promedio en la franjas horarias por hora de los dias Miercoles

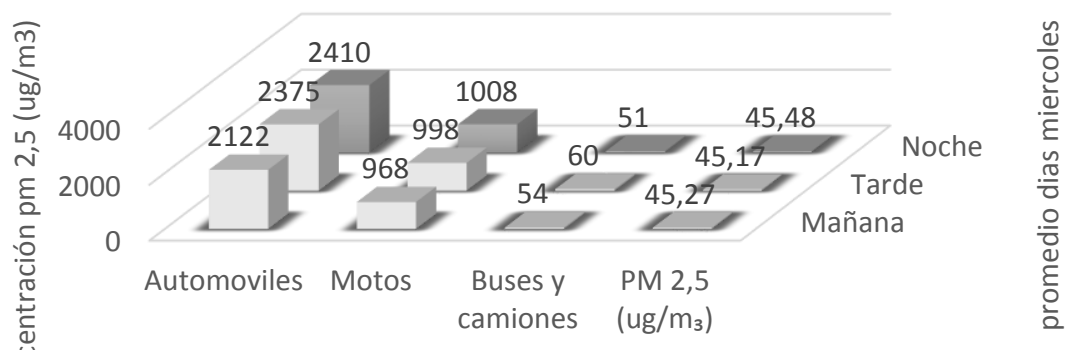

vehiculos en transito por la unab 
40

Figura 5. Medida del flujo vehicular y la concentración de PM2,5 para los lunes, martes y miércoles en las franjas: Día, tarde y noche

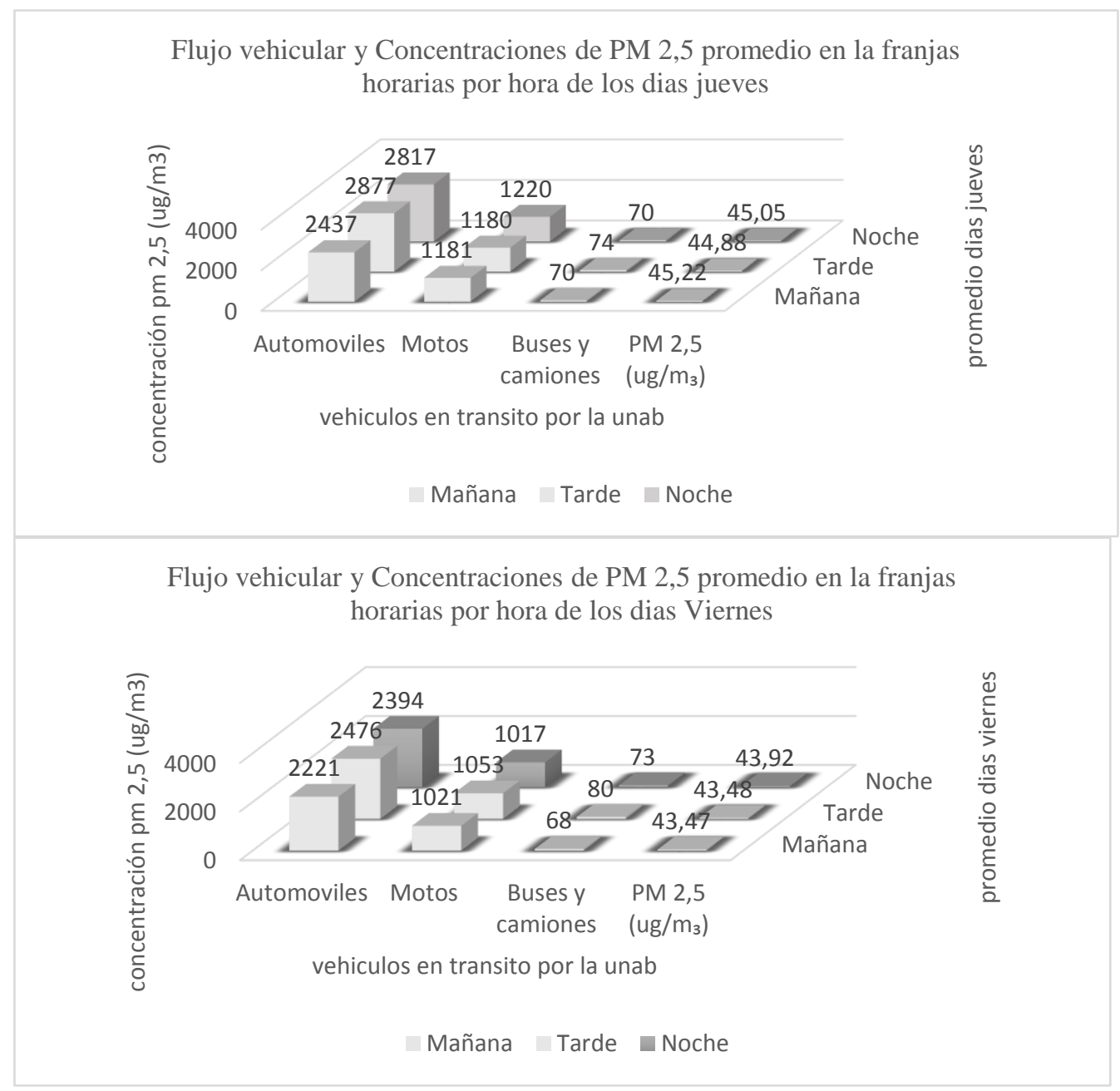


41

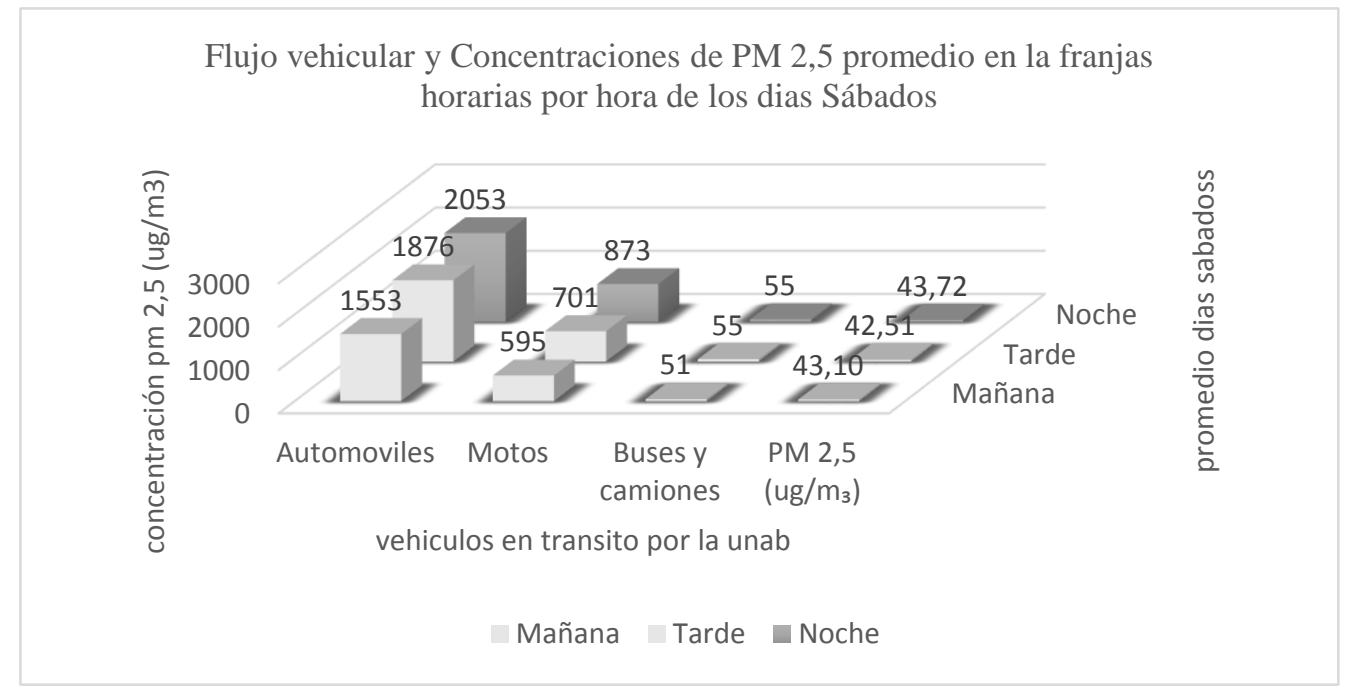

Figura 6. Medida del flujo vehicular y la concentración de PM2,5 para los jueves, viernes y sábado en las franjas: Día, tarde y noche.

L. Beleño-Montagut, Física, MSc en Física y MSc en Ingeniería ambiental de la Universidad Industrial de Santander, Bucaramanga, Colombia. Docente de la Universidad Autónoma de Bucaramanga.Actualmente líder del grupo de investigación en ciencias aplicadas (GINCAP), de la Universidad Autónoma de Bucaramanga, Colombia, el cual se encuentra en categoría C en COLCIENCIAS. ORCID: 0000-0002-6958-6367

J.D. Colegial-Gutiérrez, Geólogo. Dr. en Ciencias Geológicas de la Escuela de Minas de la Universidad Politécnica de Madrid, España. Profesor titular de la Universidad Industrial de Santander. Integrante del grupo de investigación (GIGBA) de la Universidad Industrial de Santander Bucaramanga, Colombia, el cual se encuentra en categoría A en COLCIENCIAS ORCID: 0000-0002-2320-2006

*Para citar este artículo: Beleño Montagut L.; Colegial GutierrezJ.D.Analysis of pollution by vehicular flow in a university environment. Revista Bistua.2018.16(1):28-41

+ Autor para el envió de correspondencia y la solicitud de las separatas: Beleño Montagut L. . Universidad Autónoma de Bucaramanga, Departamento de Matemáticas y Ciencias Naturales. Ibeleno@unab.edu.co

Recibido: Noviembre 18 de 2016 Aceptado:Diciembre 14 de 2017 